\author{
K.A. Bekmaganbetov ${ }^{1}$, K.Ye. Kervenev ${ }^{2}$, Ye. Toleugazy ${ }^{1}$ \\ ${ }^{1}$ M. V. Lomonosov Moscow State University, Kazakhstan Branch, Nur-Sultan, Kazakhstan; \\ ${ }^{2}$ Ye.A. Buketov Karaganda State University, Kazakhstan \\ (E-mail: toleugazy-y@yandex.ru)
}

\title{
Order of the trigonometric widths of the Nikol'skii-Besov classes with mixed metric in the metric of anisotropic Lorentz spaces
}

\begin{abstract}
In this paper we estimate the order of the triginometric width of the Nikol'skii-Besov classes $B_{\mathbf{p}}^{\alpha \tau}\left(\mathbb{T}^{n}\right)$ with mixed metric in the anisotropic Lorentz space $L_{\mathbf{q} \theta}\left(\mathbb{T}^{n}\right)$ when $\mathbf{1}<\mathbf{p}=\left(p_{1}, \ldots, p_{n}\right)<\mathbf{2}<\mathbf{q}=\left(q_{1}, \ldots, q_{n}\right)$. The concept of a trigonometric width in the one-dimensional case was first introduce by R.S. Ismagilov and he established his estimates for certain classes in the space of continuous functions. For a function of several variables exact orders of trigonometric width of Sobolev class $W_{p}^{\mathbf{r}}$, Nikol'skii class $H_{p}^{\mathbf{r}}$ in the space $L_{q}$ are established by E.S. Belinsky, V.E. Majorov, Yu. Makovoz, G.G. Magaril-Ilyaev, V.N. Temlyakov. This problem for the Besov class $B_{p q}^{\mathrm{r}}$ was investigated by A.S. Romanyuk, D.B. Bazarkhanov. The trigonometric width for the anisotropic Nikol'skii-Besov classes $B_{\mathbf{p r}}^{\alpha \tau}\left(\mathbb{T}^{n}\right)$ in the metric of the anisotropic Lorentz spaces $L_{\mathbf{q} \theta}\left(\mathbb{T}^{n}\right)$ was found by K.A. Bekmaganbetov and Ye. Toleugazy.
\end{abstract}

Keywords: trigonometric widths, anisotropic Lorentz space, Nikol'skii-Besov class with mixed metric.

\section{Introduction}

Let $V \subset L_{1}\left(\mathbb{T}^{n}\right)$ be the normed space and $F \subset V$ be some functional class. The trigonometric width of the class $F$ in the space $V$ is defined as follows (see [1])

$$
d_{M}^{T}(F, V)=\inf _{\Omega_{M}} \sup _{f \in F} \inf _{t\left(\Omega_{M} ; \mathbf{x}\right)}\left\|f(\cdot)-t\left(\Omega_{M} ; \cdot\right)\right\|_{V},
$$

where $t\left(\Omega_{M} ; \mathbf{x}\right)=\sum_{j=1}^{M} c_{j} \mathrm{e}^{i\left(\mathbf{k}_{j}, \mathbf{x}\right)}, \Omega_{M}=\left\{\mathbf{k}_{1}, \ldots, \mathbf{k}_{M}\right\}$ is the set of vectors $\mathbf{k}_{j}=\left(k_{1}^{j}, \ldots, k_{n}^{j}\right)$ from the integer lattice $\mathbb{Z}^{n}$ and $c_{j}$ are some numbers $(j=1, \ldots, M)$.

The concept of a trigonometric width in the one-dimensional case was first introduced by R.S. Ismagilov [1] and he established its estimates for certain classes in the space of continuous functions. For a function of several variables exact orders of trigonometric widths of Sobolev class $W_{p}^{\mathbf{r}}$, Nikol'skii class $H_{p}^{\mathbf{r}}$ in the space $L_{q}$ are established by E.S. Belinsky [2], V.E. Majorov [3], Yu. Makovoz [4], G.G. Magaril-Ilyaev [5], V.N. Temlyakov [6]. This problem for the Besov class $B_{p q}^{\mathrm{r}}$ was investigated by A.S. Romanyuk [7], D.B. Bazarkhanov [8]. The trigonometric width for the anisotropic Nikol'skii-Besov classes $B_{\mathbf{p r}}^{\alpha \tau}\left(\mathbb{T}^{n}\right)$ in the metric of the anisotropic Lorentz spaces $L_{\mathbf{q} \theta}\left(\mathbb{T}^{n}\right)$ was found by K.A. Bekmaganbetov and Ye. Toleugazy [9].

We study the problem of estimating the order of the trigonimetric width of the Nikol'skii-Besov classes $B_{\mathbf{p}}^{\alpha \tau}\left(\mathbb{T}^{n}\right)$ with a mixed metric in the metric of anisotropic Lorentz spaces $L_{\mathbf{q} \theta}\left(\mathbb{T}^{n}\right)$.

\section{Preliniminaries and auxiliary results}

Let $f(\mathbf{x})=f\left(x_{1}, \ldots, x_{n}\right)$ be a measurable function defined by $\mathbb{T}^{n}$. Let multiindexes $\mathbf{1} \leq \mathbf{p}=\left(p_{1}, \ldots, p_{n}\right)<$ $<\infty$. A Lebesgue space $L_{\mathbf{p}}\left(\mathbb{T}^{n}\right)$ with mixed metric is the set of functions for which the following quantity is finite

$$
\|f\|_{L_{\mathbf{p}}\left(\mathbb{T}^{n}\right)}=\left(\int_{0}^{2 \pi}\left(\ldots\left(\int_{0}^{2 \pi}\left|f\left(x_{1}, \ldots, x_{n}\right)\right|^{p_{1}} \mathrm{~d} x_{1}\right)^{p_{2} / p_{1}} \cdots\right)^{p_{n} / p_{n-1}} \mathrm{~d} x_{n}\right)^{1 / p_{n}} .
$$

Here, the expression $\left(\int_{0}^{2 \pi}|f(t)|^{p} \mathrm{~d} t\right)^{1 / p}$ for $p=\infty$ is understood as $\sup _{0 \leq t<2 \pi}|f(t)|$. 
For the function $f \in L_{\mathbf{p}}\left(\mathbb{T}^{n}\right)$ we denote

$$
\Delta_{\mathbf{s}}(f, \mathbf{x})=\sum_{\mathbf{k} \in \rho(\mathbf{s})} a_{\mathbf{k}}(f) e^{i(\mathbf{k}, \mathbf{x})},
$$

where $\left\{a_{\mathbf{k}}(f)\right\}_{\mathbf{k} \in \mathbb{Z}^{n}}$ are Fourier coefficients of the function $f$ with respect to the multiple trigonometric system $\rho(\mathbf{s})=\left\{\mathbf{k}=\left(k_{1}, \ldots, k_{n}\right) \in \mathbb{Z}^{n}: 2^{s_{i}-1} \leq\left|k_{i}\right|<2^{s_{i}}, i=1, \ldots, n\right\},(\mathbf{k}, \mathbf{x})=\sum_{j=1}^{n} k_{j} x_{j}$-inner product.

Let $\mathbf{0}<\alpha=\left(\alpha_{1}, \ldots, \alpha_{n}\right)<\infty, \mathbf{0}<\tau=\left(\tau_{1}, \ldots, \tau_{n}\right) \leq \infty$. The class of Nikol'skii-Besov $B_{\mathbf{p}}^{\alpha \tau}\left(\mathbb{T}^{n}\right)$ with a mixed metric is the set of functions $f$ from $L_{\mathbf{p}}\left(\mathbb{T}^{n}\right)$ for which the following inequality holds

$$
\|f\|_{B_{\mathbf{p}}^{\alpha \tau}\left(\mathbb{T}^{n}\right)}=\left\|\left\{\mathbf{2}^{(\alpha, \mathbf{s})}\left\|\triangle_{\mathbf{s}}(f, \cdot)\right\|_{L_{\mathbf{p}}\left(\mathbb{T}^{n}\right)}\right\}_{\mathbf{s} \in \mathbb{Z}_{+}^{n}}\right\|_{l_{\tau}} \leq 1
$$

where $\|\cdot\|_{l_{\tau}}$ is the norm of discrete Lebesgue space $l_{\tau}$ with a mixed metric.

Let $f(\mathbf{x})=f\left(x_{1}, \ldots, x_{n}\right)$ be a measurable function defined on $\mathbb{T}^{n}$. We denote by $\quad f^{*}(\mathbf{t})=f^{*_{1}, \ldots, *_{n}}\left(t_{1}, \ldots, t_{n}\right)$ the function obtained by applying to the first nonicreasing permutation, successively with respect to the variables $x_{1}, \ldots, x_{n}$ for fixed other variables.

Let multiindexes $\mathbf{q}=\left(q_{1}, \ldots, q_{n}\right), \theta=\left(\theta_{1}, \ldots, \theta_{n}\right)$ satisfy the conditions: if $0<q_{j}<\infty$, then $0<\theta_{j} \leq \infty$, if $q_{j}=\infty$, then $\theta_{j}=\infty$ for every $j=1, \ldots, n$. An anisotropic Lorentz space $L_{\mathbf{q} \theta}\left(\mathbb{T}^{n}\right)$ is the set of functions for which the following quantity is finite

$$
\|f\|_{L_{\mathbf{q} \theta}\left(\mathbb{T}^{n}\right)}=\left(\int_{0}^{2 \pi} \cdots\left(\int_{0}^{2 \pi}\left(t_{1}^{1 / q_{1}} \ldots t_{n}^{1 / q_{n}} f^{*_{1}, \ldots, *_{n}}\left(t_{1}, \ldots, t_{n}\right)\right)^{\theta_{1}} \frac{d t_{1}}{t_{1}}\right)^{\theta_{2} / \theta_{1}} \ldots \frac{d t_{n}}{t_{n}}\right)^{1 / \theta_{n}} .
$$

Let $\Omega_{M}$ be a set containing at most $M$ vectors $\mathbf{k}=\left(k_{1}, \ldots, k_{n}\right) \in \mathbb{Z}^{n}$.

Lemma 1 [10]. Let $\mathbf{2} \leq \mathbf{q}<\infty$. Then for any trigonometric polynomial

$$
P\left(\Omega_{M}, \mathbf{x}\right)=\sum_{j=1}^{M} e^{i\left(\mathbf{k}^{j}, \mathbf{x}\right)}
$$

and for any number $N \leq M$ there exists a trigonometric polynomial $P\left(\Omega_{N}, \mathbf{x}\right)$ containing at most $N$ harmonics and such that

$$
\left\|P\left(\Omega_{M}, \cdot\right)-P\left(\Omega_{N}, \cdot\right)\right\|_{L_{\mathbf{q}}\left(\mathbb{T}^{n}\right)} \leq C M N^{-1 / 2},
$$

moreover $\Omega_{N} \subset \Omega_{M}$ and all coefficients $P\left(\Omega_{N}, \mathbf{x}\right)$ are the same and do not exceed $M N^{-1}$.

Corollary 1 [11]. Let $\mathbf{2}<\mathbf{q}=\left(q_{1}, \ldots, q_{n}\right) \leq \infty, \mathbf{0}<\theta=\left(\theta_{1}, \ldots, \theta_{n}\right) \leq \infty$. Then for any trigonometric polynomial

$$
P\left(\Omega_{M}, \mathbf{x}\right)=\sum_{j=1}^{M} e^{i\left(\mathbf{k}^{j}, \mathbf{x}\right)}
$$

and for any number $N \leq M$ there exists a trigonometric polynomial $P\left(\Omega_{N}, \mathbf{x}\right)$ containing at most $N$ harmonics and such that

$$
\left\|P\left(\Omega_{M}, \cdot\right)-P\left(\Omega_{N}, \cdot\right)\right\|_{L_{\mathbf{q} \theta}\left(\mathbb{T}^{n}\right)} \leq C M N^{-1 / 2},
$$

moreover $\Omega_{N} \subset \Omega_{M}$ and all coefficients $P\left(\Omega_{N}, \mathbf{x}\right)$ are the same and do not exceed $M N^{-1}$.

For any $\mathbf{s} \in \mathbb{Z}_{+}^{n}$ we consider a linear operator

$$
\left(T_{N_{\mathbf{s}}} f\right)(\mathbf{x})=f(\mathbf{x}) *\left(\sum_{\mathbf{k} \in \rho(\mathbf{s})} e^{i(\mathbf{k}, \mathbf{x})}-t\left(\Omega_{N_{\mathbf{s}}}, \mathbf{x}\right)\right),
$$

where $t\left(\Omega_{N_{\mathbf{s}}}, \mathbf{x}\right)$ is a trigonometric polynomial from Corollary 1, which is approaching the «block» $t_{\mathbf{s}}(\mathbf{x})=\sum_{\mathbf{k} \in \rho(\mathbf{s})} e^{i(\mathbf{k}, \mathbf{x})}$.

Lemma 2. Let $\mathbf{1}<\mathbf{p}<\mathbf{2}$, the multiindex $\mathbf{q}=\left(q_{1}, \ldots, q_{n}\right)$ be such that $2<q_{j}<p^{\prime}$ for all $j=1, \ldots, n$ and $\mathbf{0}<\theta=\left(\theta_{1}, \ldots, \theta_{n}\right) \leq \infty$. Then the norm operator $T_{N_{\mathbf{s}}}$ acting from $L_{\mathbf{p}}\left(\mathbb{T}^{n}\right)$ to $L_{\mathbf{q} \theta}\left(\mathbb{T}^{n}\right)$ satisfies the following inequality

$$
\left\|T_{N_{\mathbf{s}}}\right\|_{L_{\mathbf{p}}\left(\mathbb{T}^{n}\right) \rightarrow L_{\mathbf{q} \theta}\left(\mathbb{T}^{n}\right)} \leq C_{1} 2^{(\mathbf{1}, \mathbf{s})} N_{\mathbf{s}}^{-\left(\mathbf{1} / \mathbf{2}+\mathbf{1} / \mathbf{p}^{\prime}\right)} .
$$


Proof. Taking into account that the coefficients of the polynomial $t\left(\Omega_{N_{\mathbf{s}}}, \mathbf{x}\right)$ are the same and do not exceed $2^{(\mathbf{1}, \mathbf{s})} N_{\mathbf{s}}^{-1}$ by Parseval's equality we have

$$
\left\|T_{N_{\mathbf{s}}}\right\|_{L_{\mathbf{2}}\left(\mathbb{T}^{n}\right) \rightarrow L_{\mathbf{2}}\left(\mathbb{T}^{n}\right)} \leq C_{1} 2^{(\mathbf{1}, \mathbf{s})} N_{\mathbf{s}}^{-1} .
$$

Further, using the generalized Minkowski's inequalities and Corollary 1 we can write

$$
\left\|T_{N_{\mathbf{s}}} f\right\|_{L_{\mathbf{q}^{*} \theta^{*}\left(\mathbb{T}^{n}\right)}} \leq\|f\|_{L_{1}\left(\mathbb{T}^{n}\right)}\left\|\sum_{\mathbf{k} \in \rho(\mathbf{s})} e^{i(\mathbf{k}, \cdot)}-t\left(\Omega_{N_{\mathbf{s}}}, \cdot\right)\right\|_{L_{\mathbf{q}^{*} \theta^{*}\left(\mathbb{T}^{n}\right)}} \leq C_{2} 2^{(\mathbf{1}, \mathbf{s})} N_{\mathbf{s}}^{-1 / 2}\|f\|_{L_{\mathbf{1}}\left(\mathbb{T}^{n}\right)^{\prime}} .
$$

Therefore, by definition, $\left\|T_{N_{\mathbf{s}}}\right\|_{L_{1}\left(\mathbb{T}^{n}\right) \rightarrow L_{\mathbf{q}^{*} \theta^{*}\left(\mathbb{T}^{n}\right)}}$ we find

$$
\left\|T_{N_{\mathbf{s}}}\right\|_{L_{\mathbf{1}}\left(\mathbb{T}^{n}\right) \rightarrow L_{\mathbf{q}^{*} \theta^{*}}\left(\mathbb{T}^{n}\right)} \leq C_{2} 2^{(\mathbf{1}, \mathbf{s})} N_{\mathbf{s}}^{-1 / 2}
$$

Further, using the Riesz-Thorin interpolation theorem for Lebegue spaces and anisotropic Lorentz spaces, we obtain

$$
\left\|T_{N_{\mathbf{s}}}\right\|_{L_{\mathbf{1}}\left(\mathbb{T}^{n}\right) \rightarrow L_{\mathbf{q}^{*} \theta^{*}}\left(\mathbb{T}^{n}\right)} \leq\left\|T_{N_{\mathbf{s}}}\right\|_{L_{\mathbf{2}}\left(\mathbb{T}^{n}\right) \rightarrow L_{\mathbf{2}}\left(\mathbb{T}^{n}\right)}^{1-\lambda}\left\|T_{N_{\mathbf{s}}}\right\|_{L_{\mathbf{1}}\left(\mathbb{T}^{n}\right) \rightarrow L_{\mathbf{q}^{*} \theta^{*}\left(\mathbb{T}^{n}\right)}^{\lambda},},
$$

where $\mathbf{0}<\lambda<\mathbf{1}$ and $\mathbf{1} / \mathbf{p}=(\mathbf{1}-\lambda) / \mathbf{2}+\lambda / \mathbf{1}, \mathbf{1} / \mathbf{q}=(\mathbf{1}-\lambda) / \mathbf{2}+\lambda / \mathbf{q}^{*}$ and $\mathbf{1} / \theta=(\mathbf{1}-\lambda) / \mathbf{2}+\lambda / \theta^{*}$.

By substuting (1) and (2) to (3) and performing elementary transformations, we reccieve at the required estimate with the additional condition $\mathbf{0}<\theta=\left(\theta_{1}, \ldots, \theta_{n}\right)<\mathbf{p}^{\prime}=\left(p^{\prime}, \ldots, p^{\prime}\right)$. For the remaining values of the parameters $\theta=\left(\theta_{1}, \ldots, \theta_{n}\right)$ the validy of the assertion follows from the embedding $L_{\mathbf{q} \theta_{\mathbf{1}}}\left(\mathbb{T}^{n}\right) \hookrightarrow L_{\mathbf{q} \theta_{\mathbf{2}}}\left(\mathbb{T}^{n}\right)$ for $\mathbf{0}<\theta_{1}=\left(\theta_{1}^{1}, \ldots, \theta_{n}^{1}\right) \leq \theta_{2}=\left(\theta_{1}^{2}, \ldots, \theta_{n}^{2}\right) \leq \infty$

Let us formulate a special case of the embedding theorem from E.D. Nursultanov's paper ([12]) as a Lemma.

Lemma 3 [12]. Let $\mathbf{1} \leq \mathbf{p}=\left(p_{1}, \ldots, p_{n}\right)<\mathbf{q}=\left(q_{1}, \ldots, q_{n}\right)<\infty, \mathbf{0}<\tau=\left(\tau_{1}, \ldots, \tau_{n}\right) \leq \infty$ and $\alpha=1 / p-1 / q$, then

$$
B_{\mathbf{p}}^{\alpha \tau}\left(\mathbb{T}^{n}\right) \hookrightarrow L_{\mathbf{q} \tau}\left(\mathbb{T}^{n}\right)
$$

Furthermore we need the following sets

$$
\begin{aligned}
& Y^{n}(N, \gamma)=\left\{\mathbf{s}=\left(s_{1}, \ldots, s_{n}\right) \in \mathbb{Z}_{+}^{n}: \sum_{j=1}^{n} \gamma_{j} s_{j} \geq N\right\}, \\
& \aleph^{n}(N, \gamma)=\left\{\mathbf{s}=\left(s_{1}, \ldots, s_{n}\right) \in \mathbb{Z}_{+}^{n}: \sum_{j=1}^{n} \gamma_{j} s_{j}=N\right\} .
\end{aligned}
$$

Lemma 4 [13]. Let $n \in \mathbb{N}, n \geq 2, \quad \mathbf{0}<\gamma^{\prime}=\left(\gamma_{1}^{\prime}, \ldots, \gamma_{n}^{\prime}\right) \leq \gamma=\left(\gamma_{1}, \ldots, \gamma_{n}\right)<\infty, \delta>0$ and $\mathbf{0}<\varepsilon=\left(\varepsilon_{1}, \ldots, \varepsilon_{n}\right) \leq \infty$. Then

$$
\left\|\left\{\mathbf{2}^{-\delta(\gamma, \mathbf{s})}\right\}_{\mathbf{s} \in Y^{n}\left(N, \gamma^{\prime}\right)}\right\|_{l_{\varepsilon}\left(\mathbb{Z}_{+}^{n}\right)} \leq C 2^{-\delta \eta N} N^{\sum_{j \in A \backslash\left\{j_{1}\right\}} 1 / \varepsilon_{j}},
$$

where $\eta=\min \left\{\gamma_{j} / \gamma_{j}^{\prime}: j=1, \ldots, n\right\}, A=\left\{j: \gamma_{j} / \gamma_{j}^{\prime}=\eta, j=1, \ldots, n\right\}, j_{1}=\min \{j: j \in A\}$.

Lemma 5 [13]. Let $n \in \mathbb{N}, n \geq 2, \mathbf{0}<\gamma=\left(\gamma_{1}, \ldots, \gamma_{n}\right)<\infty, \delta \in \mathbb{R}$ and $\mathbf{0}<\varepsilon=\left(\varepsilon_{1}, \ldots, \varepsilon_{n}\right) \leq \infty$. Then

$$
\left\|\left\{\mathbf{2}^{-\delta(\gamma, \mathbf{s})}\right\}_{\mathbf{s} \in \aleph^{n}\left(N, \gamma^{\prime}\right)}\right\|_{l_{\varepsilon}\left(\mathbb{Z}_{+}^{n}\right)} \asymp 2^{-\delta N} N^{\sum_{j=2}^{n} 1 / \varepsilon_{j}} .
$$

Main result

The main result of this paper includes:

Theorem 1. Let $\mathbf{1}<\mathbf{p}=\left(p_{1}, \ldots, p_{n}\right)<\mathbf{2}<\mathbf{q}=\left(q_{1}, \ldots, q_{n}\right)<\mathbf{p}_{\mathbf{0}}^{\prime}=\left(p_{0}^{\prime}, \ldots, p_{0}^{\prime}\right), p_{0}=\max \left\{p_{j}: j=\right.$ $=1, \ldots, n\}, \mathbf{1} \leq \tau=\left(\tau_{1}, \ldots, \tau_{n}\right), \theta=\left(\theta_{1}, \ldots, \theta_{n}\right)$ and $\alpha=\left(\alpha_{1}, \ldots, \alpha_{n}\right)$ be such that $\alpha_{j}>1+1 / p_{j}-1 / p_{0}$ for all $j=1, \ldots, n$. Let $\zeta=\min \left\{\alpha_{j}-1 / p_{j}+1 / q_{j}: j=1, \ldots, n\right\}, D=\left\{j=1, \ldots, n: \alpha_{j}-1 / p_{j}+1 / q_{j}=\zeta\right\}$, $j_{1}=\min \{j: j \in D\}, q_{j}=q_{j_{1}}$ for all $j \in D$ and $q_{j} \geq q_{j_{1}}$ for all $j \notin D$. 
Then the following relation holds

$$
d_{M}^{T}\left(B_{\mathbf{p}}^{\alpha \tau}\left(\mathbb{T}^{n}\right), L_{\mathbf{q} \theta}\left(\mathbb{T}^{n}\right)\right) \asymp M^{-\left(\alpha_{j_{1}}-1 / p_{j_{1}}+1 / 2\right)}(\log M)^{(|D|-1)\left(\alpha_{j_{1}}-1 / p_{j_{1}}+1 / 2\right)+\sum_{j \in D \backslash\left\{j_{1}\right\}}\left(1 / 2-1 / \tau_{j}\right)_{+}},
$$

where $|D|$ is amount of elements of the set $D, a_{+}=\max \{a ; 0\}$.

Proof. Let $f \in B_{\mathbf{p}}^{\alpha \tau}\left(\mathbb{T}^{n}\right)$. For any natural number $M$ there exists the natural number $m$ such that $M \asymp 2^{m} m^{(|D|-1)}$. We will seek an approximating polynomial $P\left(\Omega_{M}, \mathbf{x}\right)$ in the following form

$$
P\left(\Omega_{M} ; \mathbf{x}\right)=\sum_{\left(\gamma^{\prime}, \mathbf{s}\right)} \triangle_{\mathbf{s}}(f, \mathbf{x})+\sum_{m \leq\left(\gamma^{\prime}, \mathbf{s}\right)<\beta m} t\left(\Omega_{N_{\mathbf{s}}} ; \mathbf{x}\right) * \triangle_{\mathbf{s}}(f, \mathbf{x}),
$$

where

$$
\beta=\left(\alpha_{j_{1}}-1 / p_{j_{1}}+1 / 2-\frac{\log m}{m} \sum_{j \in D \backslash\left\{j_{1}\right\}}\left(\left(1 / 2-1 / \tau_{j}\right)_{+}-\left(1 / \theta_{j}-1 / \tau_{j}\right)_{+}\right)\right) /\left(\alpha_{j_{1}}-1 / p_{j_{1}}+1 / q_{j_{1}}\right),
$$

$\gamma_{j}=\left(\alpha_{j}-1 / p_{j}+1 / q_{j}\right) /\left(\alpha_{j_{1}}-1 / p_{j_{1}}+1 / q_{j_{1}}\right), j=1, \ldots, n, \gamma_{j}^{\prime}=\gamma_{j}$ for $j \in D$ and $1<\gamma_{j}^{\prime}<\gamma_{j}$ for $j \notin D$. The polynomials $t\left(\Omega_{N_{\mathbf{s}}}, \mathbf{x}\right)$ are chosen for every "block" $t_{\mathbf{s}}(\mathbf{x})=\sum_{\mathbf{k} \in \rho(\mathbf{s})} e^{i(\mathbf{k}, \mathbf{x})}$ according to Corollary 1 and numbers $N_{\mathbf{s}}=\left[2^{\left(\alpha_{j_{1}}-1 / p_{j_{1}}+1 / p_{0}\right) m} 2^{-\left(\alpha-\mathbf{1} / \mathbf{p}+\mathbf{1} / \mathbf{p}_{\mathbf{0}}-\mathbf{1}, \mathbf{s}\right)}\right]$.

Note that according to Lemma 4

$$
\begin{gathered}
\sum_{m \leq\left(\gamma^{\prime}, \mathbf{s}\right)<\beta m} N_{\mathbf{s}}=2^{\left(\alpha_{j_{1}}-1 / p_{j_{1}}+1 / p_{0}\right) m} \sum_{m \leq\left(\gamma^{\prime}, \mathbf{s}\right)<\beta m} 2^{-\left(\alpha-\mathbf{1} / \mathbf{p}+\mathbf{1} / \mathbf{p}_{\mathbf{0}}-\mathbf{1}, \mathbf{s}\right)} \leq \\
\leq 2^{\left(\alpha_{j_{1}}-1 / p_{j_{1}}+1 / p_{0}\right) m}\left\|\left\{2^{-\left(\alpha-\mathbf{1} / \mathbf{p}+\mathbf{1} / \mathbf{p}_{\mathbf{0}}-\mathbf{1}, \mathbf{s}\right)}\right\}_{\mathbf{s} \in Y^{n}\left(m, \gamma^{\prime}\right)}\right\|_{l_{1}} \leq \\
\leq 2^{\left(\alpha_{j_{1}}-1 / p_{j_{1}}+1 / p_{0}\right) m} 2^{-\left(\alpha_{j_{1}}-1 / p_{j_{1}}+1 / p_{0}\right) m} m^{(|D|-1)}=2^{m} m^{(|D|-1)} \asymp M,
\end{gathered}
$$

so that $\left(\alpha_{j}-1 / p_{j}+1 / p_{0}-1\right) /\left(\alpha_{j_{1}}-1 / p_{j_{1}}+1 / p_{0}-1\right)>\gamma_{j}^{\prime}$ at $j \notin D$.

Moreover according to equality (5) and Minkowski's inequality we have

$$
\begin{gathered}
\left\|f(\cdot)-P\left(\Omega_{M} ; \cdot\right)\right\|_{L_{\mathbf{q} \theta}\left(\mathbb{T}^{n}\right)} \leq \\
\leq C_{1}\left(\left\|\sum_{m \leq\left(\gamma^{\prime}, \mathbf{s}\right)<\beta m}\left(\triangle_{\mathbf{s}}(f, \cdot)-\triangle_{\mathbf{s}}(f, \cdot) * t\left(\Omega_{N_{\mathbf{s}}} ; \cdot\right)\right)\right\|_{L_{\mathbf{q}^{\theta}\left(\mathbb{T}^{n}\right)}}+\left\|\sum_{\left(\gamma^{\prime}, \mathbf{s}\right) \geq \beta m} \triangle_{\mathbf{s}}(f, \cdot)\right\|_{L_{\mathbf{q}^{\theta}\left(\mathbb{T}^{n}\right)}}\right)= \\
=C_{1}\left(I_{1}(f)+I_{2}(f)\right) .
\end{gathered}
$$

Firstly we estimate $I_{2}(f)$. By Lemma 3 we have

$$
I_{2}(f) \leq C_{2}\left\|\left\{2^{(\mathbf{1} / \mathbf{p}-\mathbf{1} / \mathbf{q}, \mathbf{s})}\left\|\triangle_{\mathbf{s}}(f, \cdot)\right\|_{L_{\mathbf{p}}\left(\mathbb{T}^{n}\right)}\right\}_{\mathbf{s} \in Y^{n}\left(\beta m, \gamma^{\prime}\right)}\right\|_{l_{\theta}} \leq I_{3}(f) .
$$

According to Hölder's inequality with parameters $\mathbf{1} / \theta=\mathbf{1} / \tau+\mathbf{1} / \varepsilon$, where $\mathbf{1} / \varepsilon=(\mathbf{1} / \theta-\mathbf{1} / \tau)_{+}$and Lemma 4 , taking into account that $\gamma^{\prime} \leq \gamma$ we find

$$
\begin{gathered}
I_{3}(f)=\left\|\left\{2^{(\alpha, \mathbf{s})}\left\|\triangle_{\mathbf{s}}(f, \cdot)\right\|_{L_{\mathbf{p}}\left(\mathbb{T}^{n}\right)} \cdot 2^{-\left(\alpha_{j_{1}}-1 / p_{j_{1}}+1 / q_{j_{1}}\right)(\gamma, \mathbf{s})}\right\}_{\mathbf{s} \in Y^{n}\left(\beta m, \gamma^{\prime}\right)}\right\|_{l_{\theta}} \leq \\
\leq\left\|\left\{2^{(\alpha, \mathbf{s})}\left\|\triangle_{\mathbf{s}}(f, \cdot)\right\|_{L_{\mathbf{p}}\left(\mathbb{T}^{n}\right)}\right\}_{\mathbf{s} \in Y^{n}\left(\beta m, \gamma^{\prime}\right)}\right\|_{l_{\tau}} \times \\
\times\left\|\left\{2^{-\left(\alpha_{j_{1}}-1 / p_{j_{1}}+1 / q_{j_{1}}\right)(\gamma, \mathbf{s})}\right\}_{\mathbf{s} \in Y^{n}\left(\beta m, \gamma^{\prime}\right)}\right\|_{l_{\varepsilon}} \leq
\end{gathered}
$$




$$
\begin{gathered}
\leq C_{3}\|f\|_{B_{\mathbf{p}}^{\alpha \tau}\left(\mathbb{T}^{n}\right)} \cdot 2^{-\left(\alpha_{j_{1}}-1 / p_{j_{1}}+1 / q_{j_{1}}\right) \beta m} m^{\sum_{j \in D \backslash\left\{j_{1}\right\}} 1 / \varepsilon_{j}} \leq \\
\leq C_{3} 2^{-\left(\alpha_{j_{1}}-1 / p_{j_{1}}+1 / q_{j_{1}}\right) \beta m} m^{\sum_{j \in D \backslash\left\{j_{1}\right\}}\left(1 / \theta_{j}-1 / \tau_{j}\right)_{+}} .
\end{gathered}
$$

Inserting (8) into (7) we have

$$
I_{2} \leq C_{4} 2^{-\left(\alpha_{j_{1}}-1 / p_{j_{1}}+1 / q_{j_{1}}\right) \beta m} m^{\sum_{j \in D \backslash\left\{j_{1}\right\}}\left(1 / \theta_{j}-1 / \tau_{j}\right)_{+}} .
$$

Next by using $\beta$ we obtain

$$
2^{-\left(\alpha_{\mathrm{j}_{1}}-1 / \mathrm{p}_{\mathrm{j}_{1}}+1 / \mathrm{q}_{\mathrm{j}_{1}}\right) \beta \mathrm{m}}=2^{-\left(\alpha_{\mathrm{j}_{1}}-1 / \mathrm{p}_{\mathrm{j}_{1}}+1 / 2\right) \beta \mathrm{m}} \mathrm{m}^{\sum_{\mathrm{j} \in \mathrm{D} \backslash\left\{\mathrm{j}_{1}\right\}}\left(1 / 2-1 / \tau_{\mathrm{j}}\right)_{+}},
$$

and consequently

$$
I_{2}(f) \leq C_{4} 2^{-\left(\alpha_{j_{1}}-1 / p_{j_{1}}+1 / 2\right) \beta m} m^{\sum_{j \in D \backslash\left\{j_{1}\right\}}\left(1 / 2-1 / \tau_{j}\right)_{+}} .
$$

Taking into account that $M \asymp 2^{m} m^{(|D|-1)}$ we have

$$
I_{2}(f) \leq C_{5} M^{-\left(\alpha_{j_{1}}-1 / p_{j_{1}}+1 / 2\right)}(\log M)^{(|D|-1)\left(\alpha_{j_{1}}-1 / p_{j_{1}}+1 / 2\right)+\sum_{j \in D \backslash\left\{j_{1}\right\}}\left(1 / 2-1 / \tau_{j}\right)_{+}} .
$$

Now, let us estimate the value $I_{1}(f)$. By using the Littlewood-Paley theorem (see [14]), we obtain

$$
\begin{gathered}
I_{1}(f)=\left\|\sum_{m \leq\left(\gamma^{\prime}, \mathbf{s}\right)<\beta m}\left(\triangle_{\mathbf{s}}(f, \cdot)-\triangle_{\mathbf{s}}(f, \cdot) * t\left(\Omega_{N_{\mathbf{s}}} ; \cdot\right)\right)\right\|_{L_{\mathbf{q} \theta}\left(\mathbb{T}^{n}\right)} \leq \\
\leq C_{6}\left(\left\|\sum_{m \leq\left(\gamma^{\prime}, \mathbf{s}\right)<\beta m}\left(\triangle_{\mathbf{s}}(f, \cdot)-\triangle_{\mathbf{s}}(f, \cdot) * t\left(\Omega_{N_{\mathbf{s}}} ; \cdot\right)\right)\right\|_{L_{\mathbf{q} \theta}\left(\mathbb{T}^{n}\right)}^{2}\right)^{1 / 2} \leq \\
\leq C_{6}\left\|\left\{\left\|\triangle_{\mathbf{s}}(f, \cdot) *\left(\sum_{\mathbf{k} \in \rho(\mathbf{s})} e^{i(\mathbf{k}, \cdot)}-t\left(\Omega_{N_{\mathbf{s}}}, \cdot\right)\right)\right\|_{L_{\mathbf{q} \theta}\left(\mathbb{T}^{n}\right)}\right\}_{\mathbf{s} \in \aleph^{n}\left(m, \beta m, \gamma^{\prime}\right)}\right\|_{l_{\mathbf{2}}}= \\
=C_{6}\left\|\left\{\left\|T_{\mathbf{s}} \triangle_{\mathbf{s}}(f, \cdot)\right\|_{L_{\mathbf{q} \theta}\left(\mathbb{T}^{n}\right)}\right\}_{\mathbf{s} \in \aleph^{n}\left(m, \beta m, \gamma^{\prime}\right)}\right\|_{l_{\mathbf{2}}},
\end{gathered}
$$

where $\aleph^{n}\left(m, \beta m, \gamma^{\prime}\right)=\left\{\mathbf{s} \in \mathbb{Z}_{+}^{n}: m \leq\left(\gamma^{\prime}, \mathbf{s}\right)<\beta m\right\}$.

By using Lemma 2 and inequality of different metric for trigonometric polynomials in the Lebesgue spaces with mixed metric (see [14]) for $1<p_{j}<p_{0}(j=1, \ldots, n)$, from (10) we have

$$
\begin{gathered}
I_{1}(f) \leq C_{7}\left\|\left\{2^{(\mathbf{1}, \mathbf{s})} N_{\mathbf{s}}^{-\left(1 / 2+1 / p_{0}^{\prime}\right)}\left\|\triangle_{\mathbf{s}}(f, \cdot)\right\|_{L_{\mathbf{p}_{\mathbf{0}}}\left(\mathbb{T}^{n}\right)}\right\}_{\mathbf{s} \in \aleph^{n}\left(m, \beta m, \gamma^{\prime}\right)}\right\|_{l_{\mathbf{2}}} \leq \\
\leq C_{8}\left\|\left\{2^{(\mathbf{1}, \mathbf{s})} N_{\mathbf{s}}^{-\left(1 / 2+1 / p_{0}^{\prime}\right)} 2^{\left(\mathbf{1} / \mathbf{p}+\mathbf{1} / \mathbf{p}_{\mathbf{0}}, \mathbf{s}\right)}\left\|\triangle_{\mathbf{s}}(f, \cdot)\right\|_{L_{\mathbf{p}}\left(\mathbb{T}^{n}\right)}\right\}_{\mathbf{s} \in \aleph^{n}\left(m, \beta m, \gamma^{\prime}\right)}\right\|_{l_{\mathbf{2}}}= \\
=C_{8}\left\|\left\{N_{\mathbf{s}}^{-\left(1 / 2+1 / p_{0}^{\prime}\right)} 2^{-\left(\alpha-\mathbf{1} / \mathbf{p}+\mathbf{1} / \mathbf{p}_{\mathbf{0}}-\mathbf{1}, \mathbf{s}\right)} \cdot 2^{(\alpha, \mathbf{s})}\left\|\triangle_{\mathbf{s}}(f, \cdot)\right\|_{L_{\mathbf{p}}\left(\mathbb{T}^{n}\right)}\right\}_{\mathbf{s} \in \aleph^{n}\left(m, \beta m, \gamma^{\prime}\right)}\right\|_{l_{\mathbf{2}}} .
\end{gathered}
$$

According to Hölder's inequality with parameters $\mathbf{1} / \mathbf{2}=\mathbf{1} / \tau+\mathbf{1} / \varepsilon$, where $\mathbf{1} / \varepsilon=(\mathbf{1} / \mathbf{2}-\mathbf{1} / \tau)_{+}$and by (11) we find

$$
\begin{aligned}
& I_{1}(f) \leq C_{8}\left\|\left\{2^{(\alpha, \mathbf{s})}\left\|\triangle_{\mathbf{s}}(f, \cdot)\right\|_{L_{\mathbf{p}}\left(\mathbb{T}^{n}\right)}\right\}_{\mathbf{s} \in \aleph^{n}\left(m, \beta m, \gamma^{\prime}\right)}\right\|_{l_{\tau}} \times \\
& \times\left\|\left\{N_{\mathbf{s}}^{-\left(1 / 2+1 / p_{0}^{\prime}\right)} 2^{-\left(\alpha-\mathbf{1} / \mathbf{p}+\mathbf{1} / \mathbf{p}_{\mathbf{0}}-\mathbf{1}, \mathbf{s}\right)}\right\}_{\mathbf{s} \in \aleph^{n}\left(m, \beta m, \gamma^{\prime}\right)}\right\|_{l_{\varepsilon}} \leq
\end{aligned}
$$




$$
\begin{gathered}
\leq C_{8}\|f\|_{B_{\mathbf{p}}^{\alpha \tau}\left(\mathbb{T}^{n}\right)}\left\|\left\{N_{\mathbf{s}}^{-\left(1 / 2+1 / p_{0}^{\prime}\right)} 2^{-\left(\alpha-\mathbf{1} / \mathbf{p}+\mathbf{1} / \mathbf{p}_{\mathbf{0}}-\mathbf{1}, \mathbf{s}\right)}\right\}_{\mathbf{s} \in \aleph^{n}\left(m, \beta m, \gamma^{\prime}\right)}\right\|_{l_{\varepsilon}} \leq \\
\leq C_{8}\left\|\left\{N_{\mathbf{s}}^{-\left(1 / 2+1 / p_{0}^{\prime}\right)} 2^{-\left(\alpha-\mathbf{1} / \mathbf{p}+\mathbf{1} / \mathbf{p}_{\mathbf{0}}-\mathbf{1}, \mathbf{s}\right)}\right\}_{\mathbf{s} \in \aleph^{n}\left(m, \beta m, \gamma^{\prime}\right)}\right\|_{l_{\varepsilon}}
\end{gathered}
$$

for any function $f \in B_{\mathbf{p}}^{\alpha \tau}\left(\mathbb{T}^{n}\right)$.

By continuing (12), according to the Lemma 4 we have

$$
\begin{gathered}
I_{1}(f) \leq C_{8} 2^{-\left(1 / 2+1 / p_{0}^{\prime}\right)\left(\alpha_{j_{1}}-1 / p_{j_{1}}+1 / p_{0}\right) m} \times \\
\times\left\|\left\{2^{\left(1 / 2+1 / p_{0}^{\prime}\right)\left(\alpha-\mathbf{1} / \mathbf{p}+\mathbf{1} / \mathbf{p}_{\mathbf{0}}-\mathbf{1}, \mathbf{s}\right)} \cdot 2^{-\left(\alpha-\mathbf{1} / \mathbf{p}+\mathbf{1} / \mathbf{p}_{\mathbf{0}}-\mathbf{1}, \mathbf{s}\right)}\right\}_{\mathbf{s} \in \aleph^{n}\left(m, \beta m, \gamma^{\prime}\right)}\right\|_{l_{\varepsilon}}= \\
=C_{8} 2^{-\left(1 / 2+1 / p_{0}^{\prime}\right)\left(\alpha_{j_{1}}-1 / p_{j_{1}}+1 / p_{0}\right) m} \times \\
\times\left\|\left\{2^{-\left(1 / 2+1 / p_{0}^{\prime}\right)\left(\alpha-\mathbf{1} / \mathbf{p}+\mathbf{1} / \mathbf{p}_{\mathbf{0}}-\mathbf{1}, \mathbf{s}\right)}\right\}_{\mathbf{s} \in \aleph^{n}\left(m, \beta m, \gamma^{\prime}\right)}\right\|_{l_{\varepsilon}} \leq \\
\leq C_{8} 2^{-\left(1 / 2+1 / p_{0}^{\prime}\right)\left(\alpha_{j_{1}}-1 / p_{j_{1}}+1 / p_{0}\right) m}\left\|\left\{2^{-\left(1 / 2+1 / p_{0}^{\prime}\right)\left(\alpha-\mathbf{1} / \mathbf{p}+\mathbf{1} / \mathbf{p}_{\mathbf{0}}-\mathbf{1}, \mathbf{s}\right)}\right\}_{\mathbf{s} \in Y^{n}\left(m, \gamma^{\prime}\right)}\right\|_{l_{\varepsilon}} \leq \\
\leq C_{9} 2^{-\left(1 / 2+1 / p_{0}^{\prime}\right)\left(\alpha_{j_{1}}-1 / p_{j_{1}}+1 / p_{0}\right) m} 2^{-\left(1 / 2+1 / p_{0}^{\prime}\right)\left(\alpha_{j_{1}}-\frac{1}{p_{j_{1}}}+\frac{1}{p_{0}}-1\right) m} m_{m_{j \in D \backslash\left\{j_{1}\right\}}{ }^{\sum_{\varepsilon_{j}}}}= \\
=C_{9} 2^{-\left(\alpha_{j_{1}}-1 / p_{j_{1}}+1 / 2\right) m} m^{\sum_{j \in D \backslash\left\{j_{1}\right\}}\left(1 / 2-1 / \tau_{j}\right)_{+}},
\end{gathered}
$$

as $\left(\alpha_{j}-1 / p_{j}+1 / p_{0}-1\right) /\left(\alpha_{j_{1}}-1 / p_{j_{1}}+1 / p_{0}-1\right)>\gamma_{j}^{\prime}$ at $j \notin D$.

Taking into account that $M \asymp 2^{m} m^{(|D|-1)}$ we find

$$
I_{2}(f) \leq C_{10} M^{-\left(\alpha_{j_{1}}-1 / p_{j_{1}}+1 / 2\right)}(\log M)^{(|D|-1)\left(\alpha_{j_{1}}-1 / p_{j_{1}}+1 / 2\right)+\sum_{j \in D \backslash\left\{j_{1}\right\}}\left(1 / 2-1 / \tau_{j}\right)_{+} .}
$$

Inserting (9) and (13) into (6) we obtain the inequality, which gives the upper estimate in (4).

For the proof of the lower estimate we consider the following value

$$
e_{M}(F)_{V}=\sup _{f \in F} \inf _{\left\{b_{j}, \mathbf{k}_{j}\right\}_{j=1}^{M}}\left\|f-\sum_{j=1}^{M} b_{j} e^{i\left(\mathbf{k}_{j}, \mathbf{x}\right)}\right\|_{V},
$$

which is called the best $M$-term appoximation of the class $F$ in metric space $V$.

Moreover, by the definition, the following inequality holds

$$
e_{M}(F)_{V} \leq d_{M}^{T}(F, V) \text {. }
$$

By using the condition $2<q_{j}(j=1, \ldots, n)$ we have

$$
e_{M}(f)_{L_{\mathbf{2}}\left(\mathbb{T}^{n}\right)} \leq C_{11} e_{M}(f)_{L_{\mathbf{q} \theta}\left(\mathbb{T}^{n}\right)} .
$$

For the proof of the lower estimate we will use double relation, which follows from the general results of S.M. Nikol'skii (see [15]). According to this relation for any function $f \in L_{2}\left(\mathbb{T}^{n}\right)$ the following equality holds

$$
e_{M}(f)_{L_{2}\left(\mathbb{T}^{n}\right)}=\inf _{\Omega_{M}} \sup _{P \in \mathcal{L}^{\perp},\|P\|_{L_{2}\left(\mathbb{T}^{n}\right)} \leq 1}\left|\int_{\mathbb{T}^{n}} f(\mathbf{x}) P(\mathbf{x}) d \mathbf{x}\right|,
$$

where $\mathcal{L}$ is a linear span of a system of functions $\left\{e^{i(\mathbf{k}, \mathbf{x})}\right\}_{\mathbf{k} \in \Omega_{M}}$.

We consider the function

$$
f(\mathbf{x})=m^{-\sum_{j \in D^{\prime} \backslash\left\{j_{1}\right\}} 1 / \tau_{j}} \sum_{m \leq\left(\gamma^{\prime}, \mathbf{s}_{\mathbf{0}}\right)<m+n} \prod_{j=1}^{n} 2^{-\left(\alpha_{j}+1-1 / p_{j}\right) s_{j}^{0}} \sum_{\mathbf{k} \in \rho\left(\mathbf{s}_{\mathbf{o}}\right)} e^{i(\mathbf{k}, \mathbf{x})},
$$

where $D^{\prime}=\left\{j \in D: 2<\tau_{j}\right\} \cup\left\{j_{1}\right\}, \mathbf{s}_{\mathbf{0}}=\left(s_{1}^{0}, \ldots, s_{n}^{0}\right), s_{j}^{0}=s_{j}$ at $j \in D^{\prime}$ and $s_{j}^{0}=0$ at $j \notin D^{\prime}$. 
In the paper [16] it was proved that the function $C_{12} f(\mathbf{x})$ belongs to the class $B_{\mathbf{p}}^{\alpha \tau}\left(\mathbb{T}^{n}\right)$.

Let us construct the function $P(\mathbf{x})$ satisfying the condition (14).

Let

$$
u(\mathbf{x})=\sum_{\left(\gamma^{\prime}, \mathbf{s}_{\mathbf{0}}\right) \leq m} \sum_{\mathbf{k} \in \rho\left(\mathbf{s}_{\mathbf{0}}\right)} e^{i(\mathbf{k}, \mathbf{x})},
$$

and $\Omega_{M}$ be an arbitrary collection of integer vectors $\mathbf{k}=\left(k_{1}, \ldots, k_{n}\right)$.

We denote by

$$
v(\mathbf{x})=\sum_{\left(\gamma^{\prime}, \mathbf{s}_{\mathbf{0}}\right) \leq m} \sum_{\mathbf{k} \in \rho\left(\mathbf{s}_{\mathbf{0}}\right) \cap \Omega_{M}} e^{i(\mathbf{k}, \mathbf{x})}
$$

the function, containing only those terms of (15), for which $\mathbf{k} \in \Omega_{M}$. By Minkowski's inequality and Parseval's equality for function $w(\mathbf{x})=u(\mathbf{x})-v(\mathbf{x})$ we have

$$
\|\omega\|_{L_{2}\left(\mathbb{T}^{n}\right)} \leq C_{13} M^{1 / 2}
$$

We consider the function $P(\mathbf{x})=C_{13}^{-1} M^{-1 / 2} w(\mathbf{x})$, then $\|P\|_{L_{2}\left(\mathbb{T}^{n}\right)} \leq 1$. Since the function $w(\mathbf{x})=u(\mathbf{x})-v(\mathbf{x})$ does not contain the harmonics from $\Omega_{M}$, then function $P \in \mathcal{L}^{\perp}$. Thus, the function $P(\mathbf{x})$ satisfies the conditions from (14).

According to (14) and by Lemma 5 we obtain

$$
\begin{gathered}
e_{M}(f)_{L_{2}\left(\mathbb{T}^{n}\right)} \geq C_{14} M^{-1 / 2}\left|\int_{\mathbb{T}^{n}} f(\mathbf{x}) \omega(\mathbf{x}) d \mathbf{x}\right| \geq \\
\geq C_{14} M^{-1 / 2} m^{-\sum_{j \in D^{\prime} \backslash\left\{j_{1}\right\}} 1 / \tau_{j}} \sum_{\left(\gamma^{\prime}, \mathbf{s}_{0}\right)=m} \prod_{j=1}^{n} 2^{-\left(\alpha_{j}+1-1 / p_{j}\right) s_{j}^{0}} \sum_{\mathbf{k} \in \rho\left(\mathbf{s}_{\mathbf{0}}\right)} 1= \\
\geq C_{14} M^{-1 / 2} m^{-\sum_{j \in D^{\prime} \backslash\left\{j_{1}\right\}}{ }^{1 / \tau_{j}}} \sum_{\left(\gamma^{\prime}, \mathbf{s}_{\mathbf{0}}\right)=m} \prod_{j=1}^{n} 2^{-\left(\alpha_{j}-1 / p_{j}\right) s_{j}^{0}}= \\
=C_{14} M^{-1 / 2} m^{-\sum_{j \in D^{\prime} \backslash\left\{j_{1}\right\}} 1 / \tau_{j}}\left\|\left\{2^{-\left(\alpha_{j_{1}}-1 / p_{j_{1}}\right)(\mathbf{1}, \mathbf{s})}\right\}_{\aleph^{|D|}(\mathbf{1}, \mathbf{s})}\right\|_{l_{1}} \asymp \\
\asymp M^{-1 / 2} m^{-\sum_{j \in D^{\prime} \backslash\left\{j_{1}\right\}} 1 / \tau_{j}} \cdot 2^{-\left(\alpha_{j_{1}}-1 / p_{j_{1}}\right) m} m^{(|D|-1)},
\end{gathered}
$$

where $\overline{\mathbf{s}}=\left(s_{j_{1}}, \ldots, s_{j_{|D|}}\right)$.

Taking into account that $M \asymp 2^{m} m^{(|D|-1)}$ from (16) we have

$$
\begin{gathered}
e_{M}(f)_{L_{2}\left(\mathbb{T}^{n}\right)} \geq C_{15} 2^{-\left(\alpha_{j_{1}}-1 / p_{j_{1}}+1 / 2\right) m} m^{\sum_{j \in D \backslash\left\{j_{1}\right\}}\left(1 / 2-1 / \tau_{j}\right)_{+}}= \\
=C_{16} M^{-\left(\alpha_{j_{1}}-1 / p_{j_{1}}+1 / 2\right)}(\log M)^{(|D|-1)\left(\alpha_{j_{1}}-1 / p_{j_{1}}+1 / 2\right)+\sum_{j \in D \backslash\left\{j_{1}\right\}}\left(1 / 2-1 / \tau_{j}\right)_{+} .}
\end{gathered}
$$

By (17) lower estimate in (4) follows.

Remark. Note, that for $\mathbf{p}=(p, \ldots, p), \tau=(\tau, \ldots, \tau)$ and $\mathbf{q}=\theta=(q, \ldots, q)$ the statement of the theorem coincides with the corresponding result of A.S. Romanyuk [7].

\section{Acknowledgments}

Research was partially supported by the grant of the Science Committee of Ministry of Education and Science of the Republic of Kazakhstan (grants AP05132071, AP05132590).

\section{References}

1 Ismagilov R.S. Diameter of the sets in normed linear spaces and the approximation of functions by trigonometric polynomials / R.S. Ismagilov // Russian Mathematical Surveys. - 1974. - Vol. 29, Is. 3. - P. 169-186. 
2 Белинский Э.С. Приближение периодических функций многих переменных «плавающей» системой экспонент и тригонометрические поперечники / Э.С. Белинский // Доклады АН СССР. - 1985. T. 284, № 6. - C. 1294-1297.

3 Mayorov V.E. Trigonometric diameters of the Sobolev classes $W_{p}^{r}$ in the space $L_{q} /$ V.E. Mayorov // Mathematical notes. - 1986. - Vol. 40, Is. 2. - P. 590-597.

4 Makovoz Y. On trigonometric $n$-widths and their generalization / Y. Makovoz // Journal of approximation theory. - 1984. - Vol. 41, Is. 4. - P. 361-366.

5 Магарил-Ильяев Г.Г. Тригонометрические поперечники соболевских классов функций на $R^{n} /$ Г.Г. Магарил-Ильяев // Труды МИАН СССР. - 1988. - Т. 181. - С. 147-155.

6 Temlyakov V.N. Approximations of functions with bounded mixed derivative / V.N. Temlyakov // Proc. Steklov Inst. Math. - 1989. - Vol. 178. - P. 1-121.

7 Romanyuk A.S. Kolmogorov and trigonometric widths of the Besov classes $B_{p, \theta}^{r}$ of multivariate periodic functions / A.S. Romanyuk // Sbornik: Mathematics. - 2006. - Vol. 197, Is. 1. - P. 69-93.

8 Bazarkhanov D.B. Estimates for certain approximation characteristics of Nikol'skii-Besov spaces with generalized mixed smoothness / D.B. Bazarkhanov // Doklady mathematics. - 2009. — Vol. 79, Is. 3. P. 305-308.

9 Bekmaganbetov K.A. On the order of the trigonometric diameter of the anisotropic Nikol'skii-Besov class in the metric anisotropic Lorentz spaces / K.A. Bekmaganbetov, Ye. Toleugazy // Analysis Mathematica. - 2019. - Vol. 45, Is. 2. - P. 237-247.

10 Белинский Э.С. О наименьшей величине норм смешанных производных тригонометрических полиномов с заданным числом гармоник / Э.С. Белинский, Э.М. Галеев // Вестн. МГУ. Сер. 1. Матем., мех. - 1991. - № 2. - С. 3-7.

11 Акишев Г. Оценки тригонометрических поперечников классов в пространстве Лоренца / Г. Акишев // Мат. журн. - 2012. - Т. 12, №. 4. - C. 41-57.

12 Nursultanov E.D. Interpolation theorems for anisotropic function spaces and their applications / E.D. Nursultanov // Doklady mathematics. - 2004. - Vol. 69, Is. 1. - P. 16-19.

13 Bekmaganbetov K.A. Order of the orthoprojection widths of the anisotropic Nikol'skii - Besov classes in the anisotropic Lorentz space / K.A. Bekmaganbetov, Y. Toleugazy // Eurasian Mathematical Journal. - 2016. - Vol. 7, No. 3. - P. 8-16.

14 Nikol'skii S.M. Approximation of classes of functions of several variables and embeding theorems. Springer-Verlag, Berlin, 1975. - 428 p.

15 Никольский С.М. Приближение функций тригонометрическими полиномами в среднем / С.М. Никольский // Изв. АН СССР. Сер. матем. - 1946. - Т. 10, № 3. - С. 207-256.

16 Бекмаганбетов К.А. О порядках приближения класса Бесова в метрике анизотропных пространств Лоренца / К.А. Бекмаганбетов // Уфим. матем. журн. - 2009. - Т. 1, № 2. - С. 9-16.

\author{
Қ.А. Бекмағанбетов, Қ.Е. Кервенев, Е. Төлеуғазы
}

\title{
Анизотропты Лоренц кеңістігіндегі метрикасындағы аралас метрикалы Никольский-Бесов класындағы тригонометриялық көлденеңнің реті
}

\begin{abstract}
Тригонометриялық көлденең ұғымын бірөлшемді жағдайда алғаш рет Р.С. Исмагилов енгізді және үзіліссіз функциялар кеңістігінде бірқатар кластар үшін оларға бағалаулар белгіледі. Көп айнымалы функциялар үшін $L_{q}$ кеңістігінде Соболевтің $W_{p}^{r}$, Никольскийдің $H_{p}^{r}$ кластарындағы тригонометриялық көлденеңдер үшін дәл бағалауларды Э.С.Белинский, В.Е. Майоров, Ю. Маковоз, Г.Г. МагарилИльяев, В.Н. Темляков анықтады. Бұл есепті $B_{p q}^{\mathrm{r}}$ Бесов класы үшін А.С. Романюк, Д.Б. Базарханов зерттеді. Анизотропты Никольский-Бесов $B_{\mathbf{p} r}^{\alpha \tau}\left(\mathbb{T}^{n}\right)$ класы үшін тригонометриялық көлденең анизотропты Лоренц кеңістіктері метрикасында К.А. Бекмаганбетов және Е. Төлеуғазымен табылды.
\end{abstract}

Kiлm сөздер: тригонометриялық көлденең, анизотропты Лоренц кеңістіктері, аралас метрикалы Никольский-Бесов класы. 
K.А. Бекмаганбетов, К.Е. Кервенев, Е. Толеугазы

\title{
Порядок тригонометрического поперечника класса Никольского-Бесова со смешанной метрикой в метрике анизотропного пространства Лоренца
}

\begin{abstract}
Понятие тригонометрического поперечника в одномерном случае впервые введено Р.С. Исмагиловым и им были установлены оценки для некоторых классов в пространстве непрерывных функций. Для функций многих переменных точные порядки тригонометрических поперечников класса Соболева $W_{p}^{r}$, Никольского $H_{p}^{r}$ в пространстве $L_{q}$ установлены Э.С.Белинским, В.Е. Майоровым, Ю. Маковозом, Г.Г. Магарил-Ильяевым, В.Н. Темляковым. Эта задача для класса Бесова $B_{p q}^{\mathbf{r}}$ исследована А.С. Романюком и Д.Б. Базархановым. Тригонометрический поперечник для анизотропного класса Никольского-Бесова $B_{\mathbf{p} r}^{\alpha \tau}\left(\mathbb{T}^{n}\right)$ в метрике анизотропных пространств Лоренца $L_{\mathbf{q} \theta}\left(\mathbb{T}^{n}\right)$ был найден К.А. Бекмаганбетовым и Е. Толеугазы.
\end{abstract}

Ключевые слова: тригонометрический поперечник, анизотропные пространства Лоренца, класс Никольского-Бесова со смешанной метрикой.

\section{References}

1 Ismagilov, R.S. (1974). Diameter of the sets in normed linear spaces and the approximation of functions by trigonometric polynomials. Russian Mathematical Surveys, 29, 3, 169-186.

2 Belinsky, E.S. (1985). Priblizhenie periodicheskikh funktsii mnohikh peremennykh «plavaiushchei» sistemoi eksponent i trihonometricheskie poperechniki [Approximation of periodic functions of several variables by «floating» system of exponentials, and trigonometric widths]. Doklady AN SSSR - Reports of the USSR Academy of Sciences, 284, 6, 1294-1297 [in Russian].

3 Mayorov, V.E. (1986). Trigonometric diameters of the Sobolev classes $W_{p}^{r}$ in the space $L_{q}$. Mathematical notes, 40, 2, 590-597.

4 Makovoz, Y. (1984). On trigonometric $n$-widths and their generalization. Journal of approximation theory, 41, 4, 361-366.

5 Magaril-Il'yaev, G.G. (1988). Trihonometricheskie poperechniki sobolevskikh klassov funktsii na $R^{n}$ [Trigonometric widths of Sobolev classes of function on $R^{n}$ ]. Trudy Matematicheskoho Instituta imeni V.A. Steklova - Proceedings V.A. Steklov Math. Inst., 181, 147-155 [in Russian].

6 Temlyakov, V.N. (1989). Approximations of functions with bounded mixed derivative. Proc. Steklov Inst. Math., 178, 1-121.

7 Romanyuk, A.S. (2006). Kolmogorov and trigonometric widths of the Besov classes $B_{p, \theta}^{r}$ of multivariate periodic functions. Sbornik: Mathematics, 197, 1, 69-93.

8 Bazarkhanov, D.B. (2009). Estimates for certain approximation characteristics of Nikol'skii-Besov spaces with generalized mixed smoothness. Doklady mathematics, 79, 3, 305-308.

9 Bekmaganbetov, K.A. \& Toleugazy, Y. (2019). On the order of the trigonometric diameter of the anisotropic Nikol'skii-Besov class in the metric anisotropic Lorentz spaces. Analysis Mathematica, 45, 2, $237-247$.

10 Belinskii, E.S. \& Galeev, E.M. (1991). O naimenshei velichine norm smeshannykh proizvodnykh trihonometricheskikh polinomov s zadannym chislom harmonik [On the smallest value of mixed derivatives of trigonometric polynomials with fixed number of harmonics]. Vestnik Moskovskoho universiteta. Seriia 1: Matematika. Mekhanika - Moscow University Bulletin. Ser. 1. Math., mech., 2, 3-7 [in Russian].

11 Akishev, G. (2012). Otsenki trihonometricheskikh poperechnikov klassov v prostranstve Lorentsa [Estimates of trigonometric width of classes in the Lorentz space]. Matematicheskii zhurnal - Mathematical Journal, 12, 4, 41-57 [in Russian].

12 Nursultanov, E.D. (2004). Interpolation theorems for anisotropic function spaces and their applications. Doklady mathematics, 69, 1, 16-19.

13 Bekmaganbetov, K.A. \& Toleugazy, Y. (2016). Order of the orthoprojection widths of the anisotropic Nikol'skii-Besov classes in the anisotropic Lorentz space. Eurasian Mathematical Journal, 7, 3, 8-16. 
14 Nikol'skii, S.M. (1975). Approximation of classes of functions of several variables and embeding theorems. Berlin: Springer-Verlag.

15 Nikol'skii, S.M. (1946). Priblizhenie funktsii trihonometricheskimi polinomami v srednem [Approximation of functions in the mean by trigonometrical polynomials]. Izvestiia Akademii nauk SSSR. Seriia Matematicheskaia - Izvestiia: Mathematics, 10, 3, 207-256 [in Russian].

16 Bekmaganbetov, K.A. (2009). O poriadkakh priblizheniia klassa Besova v metrike anizotropnykh prostranstv Lorentsa [About order of approximation of Besov classes in metric of anisotropic Lorentz spaces]. Ufimskii Matematicheskii zhurnal - Ufa Mathematical Journal, 1, 2, 9-16 [in Russian]. 